\title{
Produção de pós-larvas de tilápia do Nilo em sistema de recirculação de água com tanques retangulares e diferentes designs de entrada de água
}

\author{
Nile tilapia post-larvae production in a recirculating aquaculture system with rectangular \\ tanks and different water inlet designs
}

\begin{abstract}
Michael Blank de Souza (ORCID 0000-0002-0927-6850), Tuanny Trindade da Silva (ORCID 0000-0003-2815-0185), Heloise Nantes Romero Leal (ORCID 0000-0002-2338-2617), Ademar Alves Ferreira Neto (ORCID 0000-0003-3209-0007), Dacley Hertes Neu (ORCID 0000-0001-5130-773X), Cleonice Cristina Hilbig (ORCID 0000-0002-2897-6684), Vanessa Lewandowski * (ORCID 0000-0003-4946-5805)
\end{abstract}

Universidade Federal da Grande Dourados, Dourados, MS, Brasil. *Autor para correspondência: vanessalewandowski@ufgd.edu.br

Submissão: 17/05/2021 | Aceite: 13/10/2021

\begin{abstract}
RESUMO
O objetivo do presente estudo foi avaliar o efeito de diferentes formas de entrada de água em tanques retangulares na produção de pós-larvas de tilápia do Nilo em sistema de recirculação. 720 animais com peso de $0,02 \mathrm{~g}$ e comprimento de $12.52 \mathrm{~mm}$ foram distribuídos em 12 tanques retangulares, com relação de comprimento/largura de 1,20 e vazão de entrada de água de 3,2 vezes o volume útil dos tanques. Os tratamentos consistiram em três designs de entrada de água: superior, única vertical submersa e dupla vertical submersa, com quatro repetições cada. Foram avaliados o desempenho produtivo (peso e comprimento final, taxa de crescimento específico, fator de condição e sobrevivência) e a qualidade de água ( $\mathrm{pH}$, oxigênio dissolvido, temperatura, sólidos dissolvidos e condutividade elétrica). O oxigênio dissolvido foi avaliado nas quatro extremidades e ao centro de cada tanque para verificar a existência de zonas mortas. Além disso, foi verificado a concentração de sólidos suspensos, 30 minutos após o arraçoamento aos 21 dias de estudo. O design de entrada de água não influenciou no desempenho produtivo e nos parâmetros gerais de qualidade de água $(p>0,05)$. Não houve diferença na concentração de oxigênio dissolvido dentro do tanque do mesmo tratamento, indicando que não houve formação de zonas mortas $(p>0,05)$. Houve diferença na concentração de oxigênio dissolvido nas extremidades e no centro dos tanques, devido aos tratamentos, onde os menores valores foram verificados para os tanques com entrada de água única vertical submersa $(p<0,05)$. Os tanques com dupla entrada vertical submersa apresentaram menor concentração de sólidos suspensos, em comparação aos demais tratamentos $(p<0,05)$. Dessa forma, não é indicado a utilização de entrada única vertical submersa e como não houve influência negativa no desempenho produtivo e qualidade de água, é possível produzir pós-larvas de tilápia do Nilo com entrada de água superior.
\end{abstract}

PALAVRAS-CHAVE: desempenho produtivo, hidrodinâmica, qualidade de água, tanques retangulares, zonas mortas.

\begin{abstract}
The aim of the present study was to evaluate the effect of the water inlet design in rectangular tanks, on the water quality and productive performance of Nile tilapia post-larvae. 720 animals weighing $0.02 \mathrm{~g}$ and $12.52 \mathrm{~mm}$ in length were distributed in 12 rectangular tanks, with a length/width ratio of 1.20 and a water inlet flow of 3.2 times the useful volume of the tanks. The treatments consisted of three water inlet designs: upper, single vertical submerged, and double vertical submerged, distributed in four replicates each. Productive performance (weight and final length, specific growth rate, condition factor and survival) and water quality $(\mathrm{pH}$, dissolved oxygen, temperature, dissolved solids and electrical conductivity) parameter were evaluated.
\end{abstract}


Dissolved oxygen was evaluated at the four ends and center of each tank to check for dead zones. In addition, the suspended solids concentration was evaluated 30 minutes after feeding at 21 days of study. The water inlet design did not influence the productive performance and the general parameter of water quality $(p>0.05)$. There was no difference in the concentration of dissolved oxygen within the tank for each treatment, which indicates that there was no formation of dead zones $(p>0.05)$. There was a difference in the concentration of dissolved oxygen in the extremities and the center of the tanks due to the treatments, where the lowest values were verifies for the tanks with submerged vertical water inlet $(p<0.05)$. The tanks with submerged vertical double inlets showed a lower concentration of suspended solids, compared to the other treatments $(p<0.05)$. Thus, the use of a single vertical submerged inlet is not indicated and there was no negative influence on production performance and water quality, it is possible to produce Nile tilapia post-larvae with upper water inlet.

KEYWORDS: productive performance, hydrodynamics, water quality, rectangular tanks, dead zones.

\section{INTRODUÇÃO}

A aquicultura apresentou grande desenvolvimento nos últimos anos e vem sendo impulsionada pelo aumento da demanda por pescados, causada pelo crescimento populacional e pela busca de alimentos com maior qualidade nutricional. Para garantir os avanços na aquicultura é necessário otimizar os sistemas de produção a fim de melhorar os índices de desempenho dos animais durante o ciclo de vida e aumentar consequentemente o retorno econômico da atividade (FØRE et al. 2018, OBIRIKORANG et al. 2019).

Os sistemas de recirculação de água estão sendo cada vez mais usados na produção de peixes em diferentes etapas do ciclo produtivo (TIMMONS et al. 2018). O sistema fechado destinado à produção intensiva, apresenta reaproveitamento de pelo menos 90\% da água (SÁNCHEZ \& MATSUMOTO 2012). Ele proporciona uma produção eficiente e tecnificada, pois permite o controle dos parâmetros ambientais que são importantes para o desenvolvimento dos animais aquáticos (RIJN 2013).

As unidades de produção dos sistemas de recirculação são representadas por estruturas de geometria circular, hexagonal, quadrada ou retangular (OCA \& MASALÓ 2007). De acordo com OCA \& MASALÓ (2013), a hidrodinâmica no tanque é o resultado do fluxo de água, geometria da unidade de produção, entrada e saída de água. Tanques quadrados e retangulares são amplamente utilizados na aquicultura, pois proporcionam melhor aproveitamento da área, são fáceis de construir e facilitam o manejo dos peixes (OCA et al. 2004). Porém, nessas estruturas ocorre a formação de zonas mortas, que são espaços com pouca ou nenhuma troca de água, onde ocorre baixa concentração de oxigênio dissolvido e acúmulo de sólidos e metabólitos (DUARTE et al. 2011). Como os peixes evitam esses espaços, a existência de zonas mortas reduz a área útil dos tanques e resulta em menor produtividade e lucratividade do sistema de produção (GORLE et al. 2018a).

A forma de entrada influencia na velocidade e movimentação uniforme da água dentro do tanque e, consequentemente na distribuição de oxigênio dissolvido e retirada dos resíduos sólidos (BURLEY \& KLAPSIS 1985). O efeito do design de entrada na movimentação de água em tanques retangulares foi estudado por OCA et al. (2004), os quais demostraram que ocorre a formação de zonas mortas em tanques com entrada única e observa-se um padrão de fluxo circular e distribuição mais homogênea de água quando ela advém de entradas múltiplas.

Outros estudos também relatam os efeitos da geometria do tanque, fluxo, entrada e saída de água na hidrodinâmica em sistemas de recirculação (OCA \& MASALÓ 2007, OCA \& MASALÓ 2013, GORLE et al. 2018a, GORLE et al. 2019). No entanto, eles verificam a condição da água em função da engenharia do projeto, sem avaliar o efeito direto nos animais. Os fatores relacionados ao padrão de fluxo, como distribuição uniforme de oxigênio dissolvido, alimento e retirada de resíduos sólidos, são questões chaves na operação de tanques na aquicultura (GORLE et al. 2019). Eles interferem diretamente no desenvolvimento e distribuição dos peixes nas unidades de produção (DUARTE et al. 2011, OBIRIKORANG et al. 2019).

A larvicultura é uma das etapas mais críticas na aquicultura, onde verifica-se os maiores índices de mortalidade e, portanto, é necessário aprimorar as técnicas de produção com intuito de aumentar a eficiência nessa fase (EMBRAPA 2013). Nesse sentido, estudos demonstram que a hidrodinâmica exerce grande influência no desempenho alimentar de larvas de peixes e, consequentemente na sobrevivência desses organismos (CHINA \& HOLZMAN 2014, CHINA et al. 2017). 
Apesar dos estudos existentes na literatura sobre a hidrodinâmica em tanques de piscicultura, existem poucas informações sobre o efeito da entrada de água em tanques retangulares na qualidade de água e no desempenho dos peixes. Como o fornecimento de formas jovens é um fator chave para o sucesso da aquicultura, o objetivo deste estudo foi avaliar o design de entrada de água em tanques retangulares na qualidade de água e crescimento de pós-larvas de tilápia do Nilo.

\section{MATERIAL E MÉTODOS}

O estudo foi realizado no Laboratório de Aquicultura da Universidade Federal da Grande Dourados, durante 30 dias. Foram seguidas todas as diretrizes internacionais, nacionais e/ou institucionais aplicáveis para o cuidado e uso de animais (CEUA UFGD protocolo no 20.2020).

Foram utilizadas 720 pós-larvas de tilápia do Nilo com peso de $0,02 \pm 0,00 \mathrm{~g}$, pesados em balança analítica e comprimento de 12,52 $\pm 1,45 \mathrm{~mm}$, mensurado em paquímetro digital. Elas foram adquiridas de uma piscicultura comercial (Aquaforte LTDA, Dourados, MS, BR). Os animais foram distribuídos aleatoriamente em 12 tanques retangulares $(25 \mathrm{~L})$, com densidade de 3 pós-larvas $\mathrm{L}^{-1}$ ( 3000 pós-larvas $\mathrm{m}^{-3}$ ). Elas foram alimentadas com dieta comercial (Neovia LTDA, Campinas, SP, BR) com 55\% de proteína bruta, cinco vezes ao dia, até a saciedade aparente. Foi realizada a limpeza dos tanques, por meio de sifonagem, uma vez por semana, sendo retirada e posteriormente reposta, $10 \%$ do volume de água de cada unidade experimental.

O experimento foi realizado em sistema de recirculação de água, compostos por 12 tanques retangulares, um reservatório de $1000 \mathrm{~L}$, no qual localizava-se o filtro mecânico e biológico (preenchido com argila expandida). Os tanques possuíam $42,3 \mathrm{~cm}$ de comprimento e $35,0 \mathrm{~cm}$ de largura (relação de comprimento/largura de 1,20). A profundidade da água foi de $17,0 \mathrm{~cm}$, resultando em $25 \mathrm{~L}$ de volume útil. A vazão da água foi estabelecida para corresponder a $80 \mathrm{~L} \mathrm{~h}^{-1}$, o que correspondeu a 3,2 vezes o volume da unidade experimental por hora. Foi utiliza bomba centrífuga de 0,5CV com motor WEG.

$O$ estudo foi realizado com três tratamentos e quatro repetições. Os tratamentos consistiram em 3 designs de entrada de água nos tanques: superior (S), uma única vertical submersa (UV) e dupla vertical submersa (DV) (Figura 1). Para todas as formas de entrada de água, foram utilizados tubos de PVC com diâmetro de $20 \mathrm{~mm}$. A entrada superior consistia no tubo posicionado $5 \mathrm{~cm}$ dentro da unidade experimental. A única vertical submersa continha seis orifícios e foi posicionada na parede mais estreita do tanque. A dupla vertical submersa era composta por duas entradas, cada uma com três orifícios e posicionadas nas paredes maiores do tanque. Os orifícios nas entradas verticais possuíam $2 \mathrm{~mm}$ de diâmetro e foram espaçados uniformemente para cada configuração.

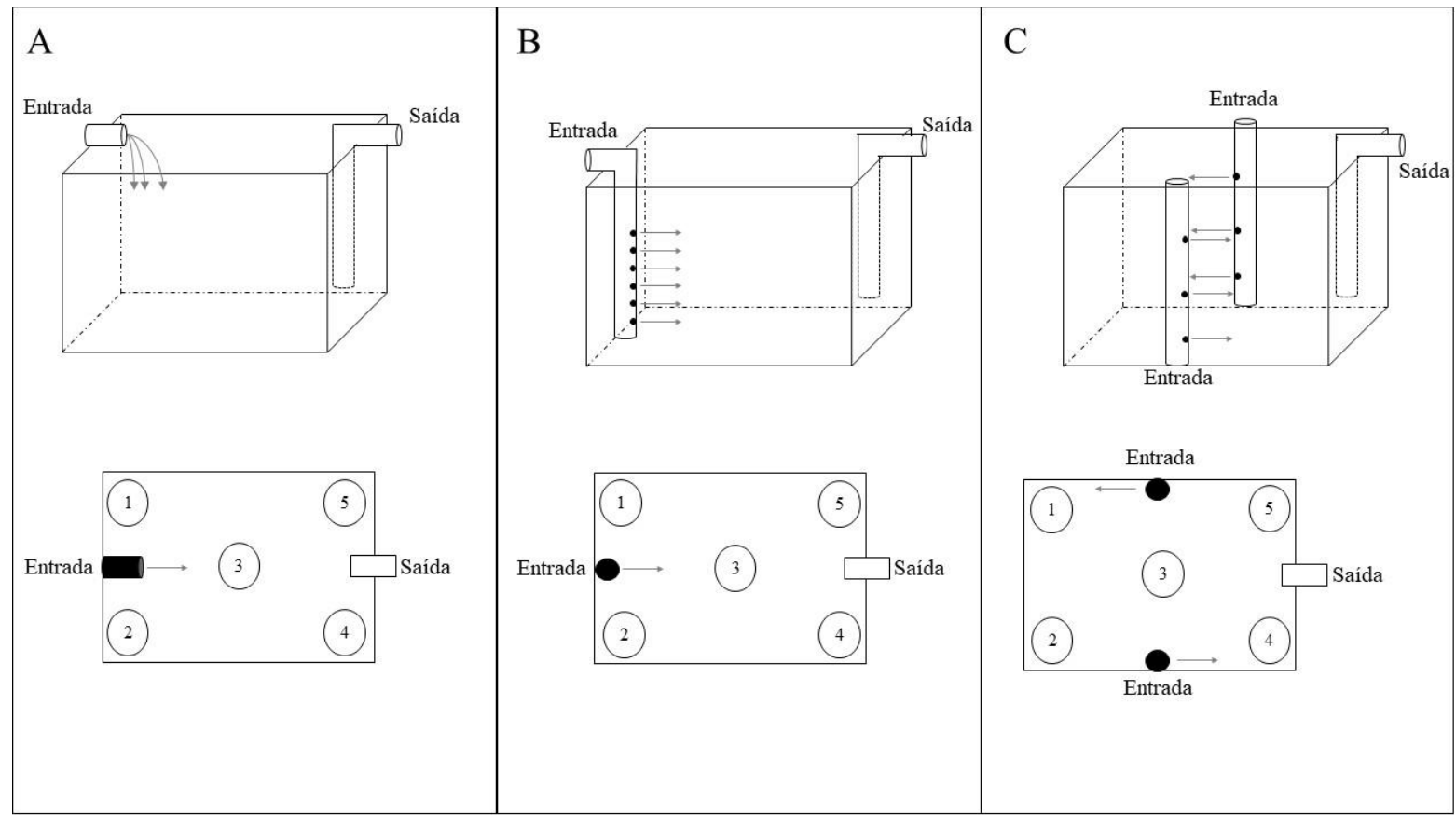

Figura 1. Design das formas de entrada de entrada de água e pontos de análise da concentração de oxigênio dissolvido para a entrada superior $(A)$, única vertical $(B)$ e dupla vertical $(C)$.

Figure 1. Design of the water inlet and the dissolved oxygen checkpoints of the upper $(A)$, single vertical $(B)$, and double vertical inlet (C). 
Ao final do período experimental, as larvas foram anestesiadas usando $150 \mathrm{mg} \mathrm{L}^{-1}$ de óleo de cravo por um minuto (RIBEIRO et al. 2015) e submetidas a uma biometria sendo verificado o peso (g) e comprimento $(\mathrm{cm})$. Por meio da biometria obteve-se os seguintes índices de desempenho produtivo:

-Comprimento final $(\mathrm{mm})$;

-Peso final (g);

-Taxa de crescimento especifico $(\mathrm{TCE})\left(\% \mathrm{dia}^{-1}\right)=\{[\ln$ peso final $(\mathrm{g})-\ln$ peso inicial $(\mathrm{g})] /$ dias experimentais\} $\times 100$.

-Fator de condição $(F C)=\left(\right.$ peso final $\times$ comprimento total/comprimento total $\left.{ }^{3}\right) \times 100$

-Sobrevivência $(S)(\%)=$ (número de indivíduos final / números de indivíduos inicial) $\times 100$.

A qualidade de água foi avaliada aos 7, 14, 21 e 28 dias de estudo. A temperatura $\left({ }^{\circ} \mathrm{C}\right)$ e o pH foram medidos com o pHmetro YSI pro 10 (YSI Inc. Yellow Springs, $\mathrm{OH}, \mathrm{USA})$. A condutividade elétrica $\left(\mu \mathrm{S} \mathrm{cm}^{-1}\right) \mathrm{e}$ sólidos dissolvidos totais $\left(\mathrm{mg} \mathrm{L}^{-1}\right)$ foram verificados com multiparâmetro digital portátil Akso AK 51 (Akso, São Leopoldo, RS, BR). O oxigênio dissolvido $\left(\mathrm{mg} \mathrm{L}^{-1}\right)$ foi mensurado com auxílio do oxímetro YSI 550A (YSI Inc.: Yellow Springs, OH, USA), em cinco pontos dos tanques, com o objetivo de verificar a influência do design da entrada de água na oxigenação em diferentes pontos do tanque, bem como a existência de zonas mortas (Figura 1).

Aos 21 dias de experimento, a concentração de sólidos suspensos totais foi determinada de acordo com a metodologia descrita por APHA (APHA 1998). A amostra de água foi coletada no centro de cada tanque 30 minutos após o arraçoamento, a fim de avaliar a influência da entrada de água na hidrodinâmica e consequente retirada de sólidos em suspensão da unidade de produção.

Os dados de desempenho produtivo e sólidos suspensos foram avaliados utilizando análise de variância (ANOVA). Os parâmetros de qualidade de água verificados semanalmente foram submetidos a análise de variância fatorial (ANOVA fatorial), onde os tratamentos e os dias de coleta foram considerados como fatores. Os dados de oxigênio dissolvido também foram avaliados separadamente, considerando cada ponto de coleta em função do tratamento, bem como se houve diferença significativa nos pontos de um mesmo tratamento. Quando os efeitos foram significados, as médias foram submetidas ao teste de Tukey com $5 \%$ de significância $(p<0,05)$. As análises estatísticas foram realizadas utilizando o software Statistica $7.0^{\circledR}$.

\section{RESULTADOS E DISCUSSÃO}

As formas de entrada de água dos tanques não influenciaram no desempenho produtivo dos animais $(p>0,05)$ (Tabela 1). O peso e comprimento final médio dos peixes do estudo foi de $1,34 \pm 0,54 \mathrm{~g}$ e $43,2 \pm$ $1,44 \mathrm{~mm}$, respectivamente. A taxa de crescimento específico permaneceu em torno de $14 \%$ e o fator de condição médio do estudo foi de $1,13 \pm 0,05$. A sobrevivência foi elevada em todos os tratamentos, permanecendo acima de $84 \%$.

Tabela 1. Desempenho produtivo de pós-larvas de tilápia do Nilo mantidas em tanques retangulares com diferentes designs de entrada de água.

Table 1. Productive performance of Nile tilapia post-larvae kept in rectangular tanks with different water inlet designs.

\begin{tabular}{lccc}
\hline & \multicolumn{3}{c}{ Designs de entrada de água } \\
\cline { 2 - 4 } & $\mathrm{S}$ & $\mathrm{DV}$ & $\mathrm{UV}$ \\
\hline Peso final $(\mathrm{g})$ & $1,35 \pm 0,54$ & $1,34 \pm 0,56$ & $1,35 \pm 0,54$ \\
Comprimento final $(\mathrm{mm})$ & $43,50 \pm 2,06$ & $42,90 \pm 0,92$ & $43,20 \pm 1,36$ \\
TCE $(\%)$ & $14,30 \pm 0,35$ & $14,30 \pm 0,24$ & $14,30 \pm 0,15$ \\
Fator de condição & $1,10 \pm 0,06$ & $1,13 \pm 0,01$ & $1,16 \pm 008$ \\
Sobrevivência $(\%)$ & $88,70 \pm 7,24$ & $90,40 \pm 8,42$ & $84,60 \pm 11,57$ \\
\hline
\end{tabular}

$\mathrm{S}=$ superior, $\mathrm{DV}=$ dupla vertical submersa, UV = única vertical submersa, TCE = Taxa de crescimento específico.

O peso e comprimento final encontrados no presente estudo foram superiores aos descritos por TACHIBANA et al. (2008), os quais obtiveram valores de $1,00 \mathrm{~g}$ e $35,00 \mathrm{~mm}$ para pós-larvas de tilápia do Nilo produzidas em densidade de 3 pós-larvas $\mathrm{L}^{-1}$. Isso demonstra que 0 ambiente aquático e a densidade de estocagem utilizada não limitaram o crescimento dos indivíduos. Animais mantidos em altas densidades reduzem a taxa de crescimento em virtude do estresse gerado no ambiente (KEBUS et al. 1992). Além disso, esses autores relatam que a elevada densidade resulta na deterioração da qualidade de água, que também afeta o crescimento dos animais. Outra questão importante no desempenho dos peixes foi a vazão 
de água utilizada, que no presente estudo correspondeu 3,2 vezes o volume do tanque por hora. Essa vazão reflete uma alta taxa de troca de água, uma vez que sistemas de recirculação comerciais operam com taxas médias de duas vezes o volume do tanque por hora (LEKANG 2013, GORLE et al. 2018b, OBIRIKORANG et al. 2019).

O baixo fluxo favorece a permanência de resíduos metabólicos no tanque e resulta em menores níveis de oxigênio na água e, consequentemente, na diminuição de ingestão de alimentos pelos peixes (OBIRIKORANG et al. 2019). Isso ocorre porque resíduos metabólicos, como fezes, favorecem o desenvolvimento de bactérias que degradam a matéria orgânica, processo no qual é realizado de forma aeróbica e com isso há aumento da demanda de oxigênio dissolvido no interior dos tanques de produção (BOYD 2012). OBIRIKORANG et al. (2019) avaliaram diferentes taxas de reposição de água de tanques no desenvolvimento e bem-estar da tilápia do Nilo e encontraram efeitos negativos no desempenho produtivo apenas para valores de troca abaixo de 3 vezes o volume do tanque por hora, em parâmetros como conversão alimentar e taxa de eficiência proteica. Já para o peso, ingestão de alimento, índices hepatossomático e viscerossomático o efeito negativo foi verificado somente para taxa de troca de água de 1,47 vezes o volume do tanque por hora.

Os parâmetros de qualidade de água permaneceram dentro das condições favoráveis para o cultivo de tilápia do Nilo (SURESH \& BHUJEL 2012). Os parâmetros de qualidade de água foram influenciados apenas em relação aos dias de avaliação $(p<0,05)$ (Figura 2$)$. Não houve diferença estatística da entrada de água ou interação entre os tratamentos e o período de avaliação $(p>0,05)$.
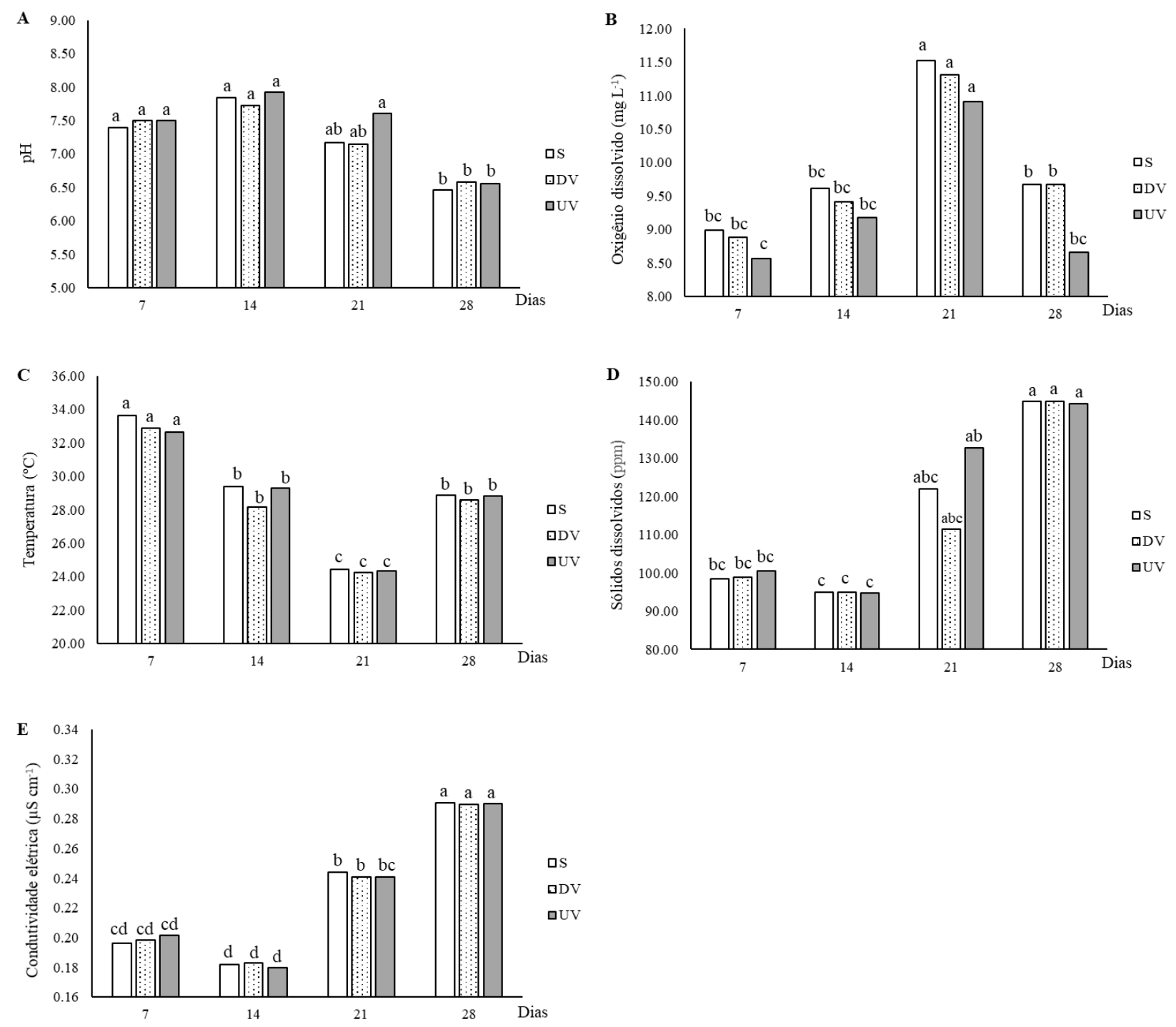

Figura 2. Qualidade de água na produção de pós-larvas de tilápia do Nilo em tanques retangulares com entrada de água superior (S), dupla vertical (DV) e única vertical (UV).

Figure 2. Water quality in the production of Nile tilapia post-larvae in rectangular tanks with upper (U), double vertical (DV), and single vertical (SV) water inlet. 
A temperatura da água variou de $24{ }^{\circ} \mathrm{C}$ a $33^{\circ} \mathrm{C}$, com valores menores aos 21 dias. Não foi aplicado sistema de controle de temperatura, a qual foi influenciada pelas mudanças da temperatura atmosférica no decorrer do estudo. A concentração de oxigênio dissolvido foi maior quando a temperatura da água foi menor, o que corrobora com os resultados descritos por LEWANDOWSKI et al. (2018). Isso ocorre pois à medida que a temperatura aumenta, a solubilidade do oxigênio diminui e a água atinge a saturação mais rapidamente (VAROL \& ŞEN 2009).

Os sólidos dissolvidos totais (SDT) e condutividade elétrica (CE) aumentaram gradualmente durante o estudo. No último dia de avaliação, os maiores valores foram registrados com as concentrações de SDT e CE de $144.75 \mathrm{mg} \mathrm{L}^{-1}$ e $0.29 \mu \mathrm{S} \mathrm{cm}^{-1}$ nos tanques com entrada superior, $144.75 \mathrm{mg} \mathrm{L}^{-1}$ e $0.28 \mu \mathrm{S} \mathrm{cm}^{-1}$ para entrada dupla vertical e $144.25 \mathrm{mg} \mathrm{L}^{-1}$ e $0.29 \mu \mathrm{S} \mathrm{cm}^{-1}$ para entrada única vertical.

A condutividade elétrica é considerada um indicador do grau de mineralização da água, pois representa a capacidade de transmissão da corrente elétrica e aumenta com a concentração iônica (BOYD 2012). Segundo esse autor, os sólidos dissolvidos totais representam partículas dissolvidas na água e são compostos por moléculas como bicarbonatos/carbonatos, cálcio, sódio, potássio e magnésio. Esses íons estão presentes na dieta e o maior tamanho dos peixes ao final do estudo refletiu no aumento da ingestão e, consequentemente, no aporte dessas moléculas para o meio aquático. Além disso, no presente estudo, foi utilizada ração farelada, o que facilita na liberação desses íons na água por meio da lixiviação, quando comparada às dietas extrusadas (SØRENSEN et al. 2009). O excesso de partículas na água afeta a saúde e bem-estar dos peixes, principalmente por danos mecânicos causados aos tecidos, como nas brânquias, que são tecidos delicados e mais susceptíveis à lesões por partículas duras derivadas de minerais (SCHUMANN \& BRINKER 2020). Nesse contexto, a condutividade elétrica é uma forma indireta de obter informações sobre a presença de partículas na água.

Independente da forma de entrada de água, não houve alteração significativa do oxigênio dissolvido dentro das unidades de produção $(p>0,05)$ e, portanto, pode-se considerar que não houve a formação de zonas mortas. No presente estudo, os tanques retangulares apresentaram relação comprimento/largura de 1,20 e segundo OCA \& MASALÓ (2007), as diferenças na hidrodinâmica são mais evidentes à partir da relação 1,91 , na qual a presença de zonas mortas é facilmente verificada.

No entanto, ao verificar os pontos de oxigênio dissolvido separadamente, houve diferença estatística em função do design de entrada de água $(p<0,05)$ (Tabela 2). A entrada de água superior resultou em maior concentração de oxigênio dissolvido nos pontos de avaliação quando comparada à única vertical submersa. Esse resultado não foi observado apenas no ponto 2 e no $7^{\circ}$ dia de execução do estudo, onde a entrada dupla vertical proporcionou maior nível de oxigênio ao comparar com a entrada única vertical submersa. As formas de entrada de água não influenciaram estatisticamente na concentração de oxigênio dissolvido no ponto 4 de avaliação nos tanques ( $p>0,05)$.

A maior concentração de oxigênio com a entrada superior favoreceu a distribuição de água na parte inicial do tanque (pontos 1 e 2), bem como, a oxigenação do centro (ponto 3). Nas extremidades posteriores (ponto 5), a concentração de oxigênio nas unidades com entrada superior também foi maior do que verificada nos tanques com entrada única vertical. Esse resultado ocorreu possivelmente pela relação comprimento/largura, ausência de zonas mortas e pelo padrão de circulação resultante da entrada única vertical submersa em tanques retangulares. Nesse design, a água sai dos orifícios com maior velocidade e é desviada para a parede do lado direito, que por sua vez, atua como um defletor e direciona a água para a parede do lado esquerdo do tanque (OCA et al. 2004). Com isso, a mistura da água nova com a água que já estava no interior dos tanques, localizada nas extremidades anteriores, posteriores e no centro, demora mais tempo para ocorrer.

Os sólidos suspensos diferiram estatisticamente entre os três designs de entrada de água $(p<0,05)$. Os tanques com entrada dupla vertical apresentaram menor concentração de sólidos suspensos 30 minutos após a alimentação. Em contrapartida, o maior valor foi encontrado em tanques com entrada de água superior, que foi 2,5 vezes maior que tanques com entrada de água dupla (Figura 3). O padrão de circulação da água em tanques retangulares com dupla entrada vertical promoveu a menor concentração de sólidos suspensos, 30 minutos após o arraçoamento, encontrado no presente estudo. Esse design promove um padrão de movimento circular e com maior velocidade, o que favorece a remoção de resíduos e a saída de água. A remoção de sólidos suspensos dos tanques é um fator importante nos sistemas de recirculação de água, pois eles causam efeitos negativos nas brânquias e podem prejudicar o desenvolvimento dos peixes (CHEN et al. 1993, GORLE et al. 2019, OBIRIKORANG et al. 2019). Apesar da diferença estatística, os valores de sólidos suspensos totais ficaram abaixo do limite estabelecido para a produção de peixes em sistemas de recirculação, que é de $100 \mathrm{mg} \mathrm{L}^{-1}$ (TIDWELL 2012). 
Tabela 2. Influência do design de entrada de água na concentração do oxigênio dissolvido.

Table 2. Influence of water inlet design on dissolved oxygen concentration.

\begin{tabular}{clccc}
\hline \multirow{2}{*}{ Pontos } & \multirow{2}{*}{ Dias } & \multicolumn{3}{c}{ Oxigênio dissolvido $\left(\mathrm{mg} \mathrm{L}^{-1}\right)$} \\
\cline { 2 - 5 } & 7 & $\mathrm{~S}$ & $\mathrm{DV}$ & $\mathrm{UV}$ \\
\hline & 14 & $8,86 \pm 0,34$ & $8,86 \pm 0,27$ & $8,75 \pm 0,36$ \\
& 21 & $9,30 \pm 0,30$ & $9,33 \pm 0,50$ & $9,06 \pm 0,48$ \\
& 28 & $11,50 \pm 0,12^{\mathrm{a}}$ & $11,30 \pm 0,33^{\mathrm{ab}}$ & $10.9 \pm 0.21^{\mathrm{b}}$ \\
& 7 & $9,72 \pm 0,77$ & $8,90 \pm 0,10$ & $8,70 \pm 0,25$ \\
\hline \multirow{2}{*}{2} & 14 & $8,83 \pm 0,21^{\mathrm{ab}}$ & $9.23 \pm 0.27^{\mathrm{a}}$ & $8.63 \pm 0.39^{\mathrm{b}}$ \\
& 21 & $9,32 \pm 0,46$ & $9,49 \pm 0,43$ & $8,97 \pm 0,29$ \\
& 28 & $11,50 \pm 0,30^{\mathrm{a}}$ & $11,4 \pm 0,36^{\mathrm{ab}}$ & $10.90 \pm 0.24^{\mathrm{b}}$ \\
& 7 & $9,82 \pm 0,72^{\mathrm{a}}$ & $8,90 \pm 0,19^{\mathrm{ab}}$ & $8.54 \pm 0.43^{\mathrm{b}}$ \\
\hline \multirow{2}{*}{3} & 14 & $8,91 \pm 0,65$ & $8,56 \pm 0,37$ & $8,73 \pm 0,21$ \\
& 21 & $10,06 \pm 0,36$ & $9,23 \pm 0,66$ & $9,28 \pm 0,22$ \\
& 28 & $11,57 \pm 0.64^{\mathrm{a}}$ & $11,28 \pm 0,18^{\mathrm{ab}}$ & $10,93 \pm 0.23^{\mathrm{b}}$ \\
& 7 & $9,72 \pm 0,64$ & $8,91 \pm 0,18$ & $8,73 \pm 0,28$ \\
\hline & 14 & $9,09 \pm 0,60$ & $9,03 \pm 0,35$ & $8,42 \pm 0,29$ \\
& 21 & $9,69 \pm 0,45$ & $9,57 \pm 0,74$ & $9,30 \pm 0,23$ \\
& 28 & $11,51 \pm 0,54$ & $11,52 \pm 0,18$ & $10,83 \pm 0,23$ \\
& 7 & $9,60 \pm 0,73$ & $8,94 \pm 0,07$ & $8,70 \pm 0,30$ \\
\hline \multirow{2}{*}{5} & 14 & $9,22 \pm 0,33^{\mathrm{a}}$ & $8,74 \pm 0,34^{\mathrm{ab}}$ & $8.30 \pm 0.54^{\mathrm{b}}$ \\
& 21 & $9,68 \pm 0,61$ & $9,45 \pm 0,68$ & $9,28 \pm 0,24$ \\
& 28 & $11,51 \pm 0,47$ & $11,03 \pm 0,18$ & $11,08 \pm 0,67$ \\
& 28 & $9,50 \pm 0,67$ & $8,68 \pm 0,20$ & $8,62 \pm 0,40$ \\
\hline
\end{tabular}

$\mathrm{S}=$ superior, $\mathrm{DV}=$ dupla vertical submersa, $\mathrm{UV}=$ única vertical submersa. Letras minúsculas indicam diferença estatística na mesma linha, pelo teste de Tukey $(p<0,05)$.

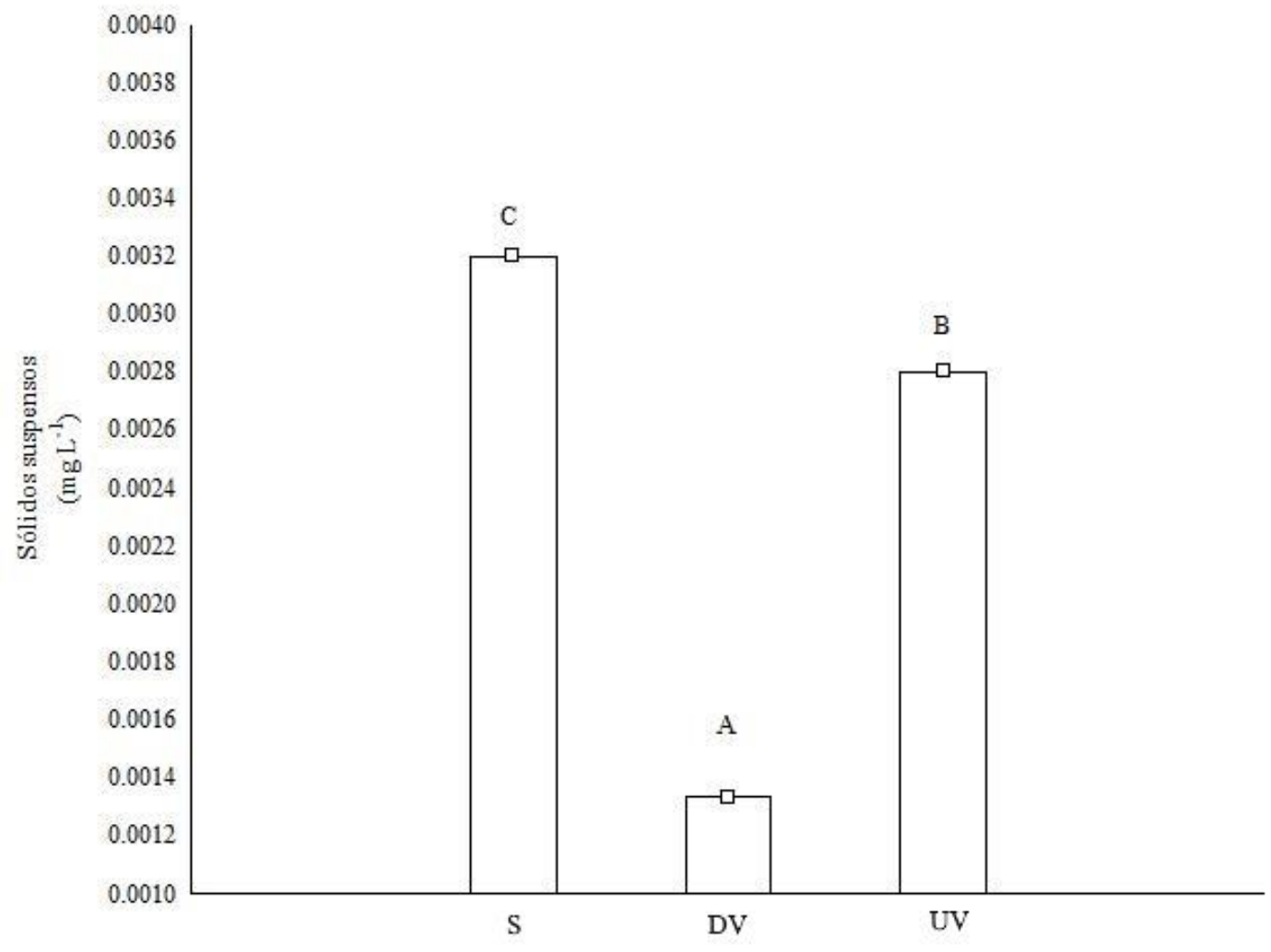

Figura 3. Concentração de sólidos suspensos no interior de tanques retangulares 30 minutos após o arraçoamento de pós-larvas de tilápia do Nilo. $\mathrm{S}$ = entrada de água superior, DV = entrada de água dupla vertical, UV = entrada de água única vertical. Letras distintas indicam diferença significativa pelo teste de Tukey $(p<0,05)$.

Figure 3. Suspended solids concentrations inside rectangular tanks 30 minutes after the feeding Nile tilapia post-larvae. $S=$ upper water inlet, $D V=$ double vertical inlet, $U V=$ single vertical water inlet. Different letters indicate a significant difference by the Tukey test $(p<0.05)$. 


\section{CONCLUSÃO}

A entrada de água superior não prejudica a qualidade de água e o desempenho produtivo e, portanto, pode ser utilizada na produção de pós-larvas de tilápia do Nilo em tanques retangulares, com relação comprimento/largura de 1,20 e vazão correspondente a 3,2 vezes do seu volume útil por hora. Devido a influência na concentração de oxigênio dissolvido, não é indicado a utilização de entrada de água única vertical.

\section{AGRADECIMENTOS}

À Universidade Federal da Grande Dourados (UFGD), pelo aporte de recursos financeiros que custearam a pesquisa e a empresa Aquaforte LTDA pela doação dos animais utilizados na execução do estudo.

\section{REFERÊNCIAS}

APHA. 1998. Standard Methods for the Examination of Water and Wastewater. 20.ed. Washington: American Water Works Association and Water Environmental Federation.

BOYD CE. 2012. Water quality. In: LUCAS JS \& SOUTHGATE PC (Ed.). Aquaculture: farming aquatic animals and plants. Chichester: Blackwell Publishing. p.52-82.

BURLEY R \& KLAPSIS A. 1985. Flow distribution studies in fish rearing tanks. Part 2-analysis of hydraulic performance of $1 \mathrm{~m}$ square tanks. Aquacultural Engineering 4: 113-134.

CHEN S et al. 1993. Suspended solids characteristics from recirculating aquacultural systems and design implications. Aquaculture 112: 143-155.

CHINA V et al. 2017. Hydrodynamic regime determines the feeding success of larval fish through the modulation of strike kinematics. Proceedings of the Royal Society B: Biological Sciences 284: 1-8.

CHINA V \& HOLZMAN R. 2014. Hydrodynamic starvation in first-feeding larval fishes. Proceedings of the National Academy of Sciences of the United States of America 111: 8083-8088.

DUARTE S et al. 2011. Influence of tank geometry and flow pattern in fish distribution. Aquacultural Engineering 44: 4854.

EMBRAPA. 2013. Piscicultura de água doce: multiplicando conhecimentos. 1.ed. Brasília: Embrapa.

FØRE $M$ et al. 2018. Precision fish farming: a new framework to improve production in aquaculture. Biosystems Engineering 173: 176-193.

GORLE JMR et al. 2018a. Hydrodynamics of octagonal culture tanks with Cornell-type dual-drain system. Computers and Electronics in Agriculture 151: 354-364.

GORLE JMR et al. 2018b. Water velocity in commercial RAS culture tanks for Atlantic salmon smolt production. Aquacultural Engineering 81: 89-100.

GORLE JMR et al. 2019. Hydrodynamics of Atlantic salmon culture tank: Effect of inlet nozzle angle on the velocity field. Computers and Electronics in Agriculture 158: 79-91.

KEBUS MJ et al. 1992. Effects of rearing density on the stress response and growth of rainbow trout. Journal of Aquatic Animal Health 4: 1-6.

LEKANG OI. 2013. Aquaculture Engineering. 2.ed. Oxford: John Wiley \& Sons.

LEWANDOWSKI V et al. 2018. Spatial and temporal limnological changes of an aquaculture area in a neotropical reservoir. Annales de Limnologie 54: 1-9.

OBIRIKORANG KA et al. 2019. Effects of water flow rates on growth and welfare of Nile tilapia (Oreochromis niloticus) reared in a recirculating aquaculture system. Aquaculture International 27: 449-462.

OCA J \& MASALÓ I. 2013. Flow pattern in aquaculture circular tanks: Influence of flow rate, water depth, and water inlet and outlet features. Aquacultural Engineering 52: 65-72.

OCA J \& MASALÓ I. 2007. Design criteria for rotating flow cells in rectangular aquaculture tanks. Aquacultural Engineering 36: 36-44.

OCA $J$ et al. 2004. Comparative analysis of flow patterns in aquaculture rectangular tanks with different water inlet characteristics. Aquacultural Engineering 31: 221-236.

RIBEIRO PAP et al. 2015. Efficiency of eugenol as anesthetic for the early life stages of Nile tilapia (Oreochromis niloticus). Anais da Academia Brasileira de Ciencias 87: 529-535.

SÁNCHEZ IA \& MATSUMOTO T. 2012. Hydrodynamic characterization and performance evaluation of an aerobic three phase airlift fluidized bed reactor in a recirculation aquaculture system for Nile Tilapia production. Aquacultural Engineering 47: 16-26.

SCHUMANN M \& BRINKER A. 2020. Understanding and managing suspended solids in intensive salmonid aquaculture: a review. Reviews in Aquaculture 12: 2109-2139.

SØRENSEN M et al. 2009. Soybean meal improves the physical quality of extruded fish feed. Animal Feed Science and Technology 149: 149-161.

SURESH V \& BHUJEL RC. 2012. Tilapias. In: LUCAS JS \& SOUTHGATE PC. (Ed.). Aquaculure: farming aquatic animals and plants. Chichester: Blackwell Publishing Ltd.p.338-383.

TACHIBANA L et al. 2008. Densidade de estocagem de pós-larvas de tilápia do Nilo (Oreochromis niloticus) durante a 
fase de reversão sexual. Boletim do Instituto de Pesca 34: 483-488.

TIDWELL JH. 2012. Aquaculture production systems. 1.ed. Chichester: Blackwell Publishing.

TIMMONS et al. 2018. Recirculating Aquaculture. 4.ed. New York: Ithaca Publishing Company, 2018.

RIJN JV. 2013. Waste treatment in recirculating aquaculture systems. Aquacultural Engineering 53: 49-56.

VAROL M \& ŞEN B. 2009. Assessment of surface water quality using multivariate statistical techniques: a case study of Behrimaz Stream, Turkey. Environmental Monitoring and Assessment 159: 543-553. 